Conclusion Both documents are concrete steps towards promoting sexual health as a public health concept at international and national levels.

\section{S03.2 SEXUAL HEALTH PROMOTION INTERVENTIONS: RESULTS OF A SYSTEMATIC REVIEW}

doi:10.1136/sextrans-2013-051184.0021

M S Hogben, J V Ford, J Becasen. Centers for Disease Control \& Prevention, Atlanta, GA, United States

Background Population-level rates of sexual health indicators such as STI rates have stimulated interest in a public health approach to improving sexual health in the United States. We used several existing definitions (World Health Organization, U.S Surgeon-General's office, CDC/HRSA Advisory Committee) to derive sexual health principles: recognition of sexuality as intrinsic to individual health and relationships should have positive outcomes for all partners involved.

Methods Studies for a systematic review of intervention literature were drawn from Medline and PsycInfo databases (English language, adult populations, published between 1996-2011, country with developed public health infrastructure). They addressed outcomes in one or more domains: knowledge, attitudes, communication, healthcare use, sexual behaviours or adverse events. Data were summarised in a narrative review organised by population (adults, parents, sexual minorities, vulnerable populations) across domains. Selected data from knowledge, attitudes and behaviours were summarised in meta-analyses.

Results From 9064 studies, 58 were retained in the narrative review. Studies employed qualitative, experimental, pre-post and matched comparison group designs; the number of studies published was correlated with publication year $(r=0.77, p<0.001)$. Interventions were predominantly individual and small-group in-person designs that addressed sexual behaviours (42 studies, $72 \%$ ) and attitudes/norms $(32,55 \%)$. Studies with parents covered communication. All but one study reported at least one positive finding, but many (29 studies, $50 \%$ ) also reported null findings. The most consistent positive effects on behaviours and adverse events were found for sexual minorities and vulnerable populations; interventions with parents uniformly increased attitudes and communication skills.

Conclusions Sexual health-framed interventions generate positive effects across adult populations, as well as mental and behavioural domains and adverse outcomes. Interventions may be especially effective among vulnerable populations and in improving parent communication. Where scalable, incorporating aspects of existing sexual health definitions into public health may contribute to improving sexual health.

\section{S03.3 ADDRESSING GENDER-BASED VIOLENCE TO REDUCE RISK OF STI AND HIV}

doi:10.1136/sextrans-2013-051184.0022

A. Amin, C. Garcia Moreno, World Health Organization, Department of Reproductive Health and Research, Geneva, Switzerland.

Background Gender-based violence, and gender inequality more broadly, has been found to be associated with increased risk of sexually transmitted infections (STI) including HIV among women and girls as well as among key vulnerable groups such as sex workers. This paper presents the evidence of the increased risk of STI and HIV associated with gender-based violence; and looks at potential pathways by which gender-based violence and STI and HIV are linked.

Methods A systematic review and meta-analysis of studies that measure the association between intimate partner violence and STI and HIV was conducted by the London School of Hygiene and Trop- ical Medicine and WHO as part of work feeding into the Global Burden of Disease Study estimates on violence against women and its health impacts. Another systematic review of studies that measure association between violence against sex workers and STI and HIV was also conducted by the University of British Columbia, Vancouver and WHO. Other studies and literature were reviewed to identify potential pathways to explain the links between genderbased violence and HIV.

Results The results of the systematic review show that best estimate of association between physical and/or sexual intimate partner violence and HIV was an odds ratio (OR) of 1.52 (95\% CI $=1.03$ to 2.23) for HIV, from studies from generalized and concentrated HIV epidemics and slightly higher for syphilis, chlamydia or gonorrhea. . These studies, however, are mainly cross sectional population-based surveys among women in the general population. The systematic review of violence against sex workers shows that sex workers from India and US who experience sexual violence have between 2 and 3 -fold increased risk of HIV sero-positivity. Sex workers who experience any form of physical or sexual violence by any perpetrator in studies from India (Karnataka), Thailand, USA (San Francico) also showed increased risk of STI sero-positivity. Studies suggest 4 potential pathways linking gender-based violence and STI/HIV. First, sexual violence can be directly associated with increased STI and HIV transmission. There are also several indirect mechanisms; these include a history of violence in childhood or adolescence being linked to increased sexual risk taking later; and difficulties in negotiation of condom use with the partner. Also, men who perpetrate violence are also more likely to engage in sexual risk taking. Third, fear of violence can prevent women and sex workers from seeking or accessing HIV information and services. Lastly, violence can be an outcome of diagnosis and disclosure of HIV status.

Conclusion Interventions to address the HIV epidemic among women and among sex workers need to address violence as a risk factor. In each setting, interventions need to be based on an understanding of the potential pathways that link violence against women and sex workers to STI and HIV infection. HIV prevention, treatment, and care programmes for women and for sex workers can integrate violence prevention into their risk-reduction counselling and communication, work with men and boys to promote gender equality and reduce violence perpetration, empower women, girls and sex workers, address harmful gender norms that perpetuate the acceptability of violence, and address the harmful use of alcohol. Laws and policies that criminalize sex workers and that perpetuate gender-based discrimination against women and girls also need to be addressed.

\section{S03.4 SEXUAL HEALTH IN THE EUROPEAN UNION: AN INVENTORY OF DATA, PROGRAMMES, INITIATIVES, AND POLICIES}

doi:10.1136/sextrans-2013-051184.0023

\section{Van de Laar, 0 Sfetcu. ECDC, Stockholm, Sweden}

Background Sexually transmitted infections and HIV represent a major public health challenge in European Union/European Economic Area. In 2011, 346911 chlamydia, 39179 gonorrhoea, 28038 HIV and 19798 syphilis cases were reported to the European Centre for Disease Prevention and Control (ECDC). Improving prevention efforts is therefore a priority, especially among populations groups that are most vulnerable to infection, including young people, MSM, migrants and people living with HIV/AIDS

Methods A mapping exercise of European sexual health data, programmes, initiatives and policies was conducted aiming to increase the understanding of how HIV/STI can be prevented in the general population and among vulnerable population groups. 